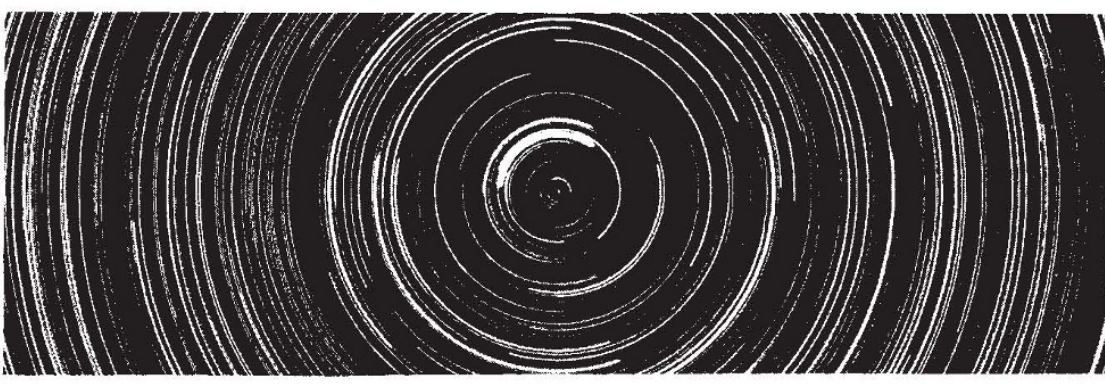

The Earth moved - star tracks on an 8-hour exposure taken by a fixed camera pointed towards the North Pole; the boldest trail near the centre was made by Polaris. The picture is taken from the new paperback edition of Orbiting the Sun: Planets and Satellites of the Solar System by Fred Whipple (reviewed in Nature 294,$21 ; 1981$ ). Publisher is Harvard University Press, price is $\$ 9.25, £ 6.75$.

tions (hardly surprising). In fact, the coverage is much more diverse than this with treatments of topics not conventionally associated with the books' title, for instance design, safety and health, materials and machines in antiquity, materials economics and management, art materials, newsprint and many materials of biological origin.

The Systematic Outline of EMSE (see Vols 1 and 8) groups the article titles under the 45 main subject headings used in the commissioning; they cover the production of materials, their nature and applications, property and structure assessment techniques, surface phenomena and processes, and more general topics such as mineral resources and management, and economics. These are further subdivided "as each subject dictates". The Introduction states that the space devoted to each of these sub-sections is in relation to its scientific, technical and industrial importance but we are given little insight into how this was assessed. The basis of selection must, in the end, be subjective and the updating policy will show whether the editors have met their goals.

The 1,570 articles are arranged alphabetically, which is a rather mixed blessing. This format should force users to look outside their specialities and, one hopes, help the editors to achieve their aim of cross-fertilization. The juxtaposition of "Titanium Alloys" with "Timbers from Various Geographical Locations" and "The Effect of Tax Upon Materials Policy" demonstrates this amply. On the other hand, the scheme puts a lot of stress on the cross-referencing system and it doesn't hold up too well, especially in terms of alternative titles (for example, "glassy" and "amorphous"). In addition, because the articles are not grouped under, say, specific materials, properties, processes or artefacts, the subject index - which is in Vol. 8 and very thorough - is an absolute must if you are looking for something specific.

Basically, EMSE consists of two types of entry: specific articles on particular topics in the science and engineering of materials, and articles of a more general kind. By and large the specific articles are authoritative, if succinct, treatments of their subjects but there is considerable variation in the level and approach. Perhaps this is inevitable, given the need to involve so many different authors each with their own ideas about the task, but there does seem to have been a certain unwillingness either to give clear briefing or to wield the editorial pen in the quest for consistency.

It is with the general articles that EMSE is clearly trying to be more than a conventional encyclopaedia. These are either reviews (for example "Electrical Materials - An Overview" and "Energy Considerations in Metals Production") or articles of an integrative nature ("Ceramics: Relation of Microstructure to Processing History", "Product Liability and Materials" and "Design and Materials Selection and Processing", for instance). The integrative articles are particularly informative and stimulating; they are good illustrations of the way to link aspects of materials that are often treated in isolation and, therefore, inadequately. Their inclusion is a clear manifestation of the editors' laudable desire to lower the barriers between disciplines. But beware, you will not find these gems in the subject index - for these you will need to use the Systematic Outline (Vol. 8).

In addition to unevenness in the level of the articles, the work can also be criticized for its slight bias towards the United States, and for a hint of partisanship. However, at this early stage in what should be an evolutionary process for EMSE, we will call this carping and trust that the balance is adjusted in the next edition.

The editors have tackled an enormous challenge and have met with considerable success. These volumes will be an important reference source for all scientists and engineers involved with the properties, processing and uses of materials, and especially for teachers of courses in the materials field.

Charles Newey, Mark Endean, Peter Lewis, Nick Reid, Graham Weaver, George Weidmann and John Wood are members of the Materials Discipline, The Open University, Walton Hall, Milton Keynes MK7 6AA, UK.

\section{Living for physics}

\author{
Michael Holwill
}

Introductory Biophysics. By M. Cerdonio and R.W. Noble. World Scientific: 1986. Pp.216. £20.25, \$25.30.

FOR those who browse among the shelves of libraries or bookshops, the title of this book and the attractive seascape on the dust-jacket will provide little indication of the contents. It is not an introductory text for students embarking on a course in biophysics; rather, it is aimed at scientists with a reasonable background in physics, particularly in thermodynamics, and it is intended to demonstrate that the basis of living systems can be understood and explained by the application of physical principles. This is not a new concept, but recent developments in both molecular cell biology and irreversible thermodynamics make it appropriate to present a modern account of the ideas concerned.

After a brief description of the characteristics by which living systems can be identified, and a chapter dealing with the structure and organization of those systems, from viroids through bacteria to multicellular organisms, the authors apply physical ideas to a number of biological areas before discussing, in the final chapter, the origins of life. The chapters are essentially independent, each containing some interesting features but having little direct relationship to the others. There is, in fact, much of importance and value in the book, but the rather dated diagrams (which vary considerably in style and quality) and the use of old-fashioned units (for example, "entropy units" instead of $\mathrm{J} \mathrm{K}^{-1}$ $\mathrm{mol}^{-1}$ and "calories" for joules) detract from the modern scientific approach.

It is convenient to have, collected together in a single volume, accounts of the essentials of various physico-chemical processes and techniques relevant to a comprehensive examination of the nature and development of living systems. By reducing the space occupied by some of the diagrams, however, a little more introductory material could have been added without the need for additional pages, and this would have increased the appeal of the work to the great number of scientists who have a general interest in this controversial topic. In the final chapter the authors provide some pertinent insights into how thermodynamics, chemical kinetics and fundamental particle physics may be of importance in specifying evolutionary processes, but they have not capitalized on the material earlier in the book by giving a clear summary of the current status of this analytical study.

Michael Holwill is Reader in Biophysics in the Department of Physics, King's College London (KQC), Strand, London WC2R $2 L S, U K$. 